\title{
On the Jebsen-Birkhoff Theorem in a Born-Infeld Type Theory of Gravity
}

\author{
Tiago de Oliveira Rosa ${ }^{1}$, Maria Emilia Xavier Guimarães ${ }^{2}$, Rocco Pascale Neto ${ }^{2}$, \\ Joaquim Lopes Neto ${ }^{3}$
}

\author{
${ }^{1}$ Instituto Federal Goiano, Campus Urutaí, Rodovia Geraldo Silva Nascimento, Urutaí, Brazil \\ ${ }^{2}$ Instituto de Física, Universidade Federal Fluminense, Niterói, Brazil \\ ${ }^{3}$ Instituto de Física, Universidade Federal do Rio de Janeiro, Rio de Janeiro, Brazil \\ Email: tiago.rosa@ifgoiano.edu.br, emilia@if.uff.br, roccopascale@id.uff.br, jlneto@if.ufrj.br
}

\begin{abstract}
How to cite this paper: de Oliveira Rosa, T., Guimarães, M.E.X., Pascale Neto, R. and Neto, J.L. (2019) On the Jebsen-Birkhoff Theorem in a Born-Infeld Type Theory of Gravity. Journal of High Energy Physics, Gravitation and Cosmology, 5, 1051-1056.
\end{abstract}

https://doi.org/10.4236/jhepgc.2019.54057

Received: August 14, 2019

Accepted: September 15, 2019

Published: September 18, 2019

Copyright (C) 2019 by author(s) and Scientific Research Publishing Inc.

This work is licensed under the Creative Commons Attribution International License $(\mathrm{CC}$ BY 4.0).

http://creativecommons.org/licenses/by/4.0/

\begin{abstract}
In this paper we work with a special theory of gravity - the NovelloDi Lorenci-Luciane (hereby called NDL theory) which extends the Feynman-Deser standard theoretical-field approach to General Relativity. In the so-called NDL theory, matter interacts universally with gravity in accordance with the Weak Equivalence Principle, while gravitons have a nonlinear self-interaction. Our main aim in this work is to show that, though the NDL theory does not admit a Schwarzschild solution, the Jebsen-Birkhoff theorem is still valid in this framework.
\end{abstract}

\section{Keywords}

Electrostatic Trapping, Bootstrap Current, Mitigation, Peeling-Ballooning Mode

\section{Open Access}

\section{Introduction}

Although there exist many effective theories of gravity which come from the unification process $[1,2]$, each of them must, of course, satisfy some predictions. Actually, with the advent of the recent LIGO detections could be, in principle, an important and useful tool to test the alternative theories of gravity as it has been pointed out by Corda in Ref. [3]. Here, in this paper, we will deal with one of the extended gravity theory, the so-called NDL theory $[4,5]$ which is based on a Born-Infeld theory [6].

Inspired by the great issue of the Born-Infeld program [6] concerning avoiding singularities, some generalizations of gravitational BornInfeld type lagrangians have been made [7]. In particular, it has been shown that singularities like the Schwarszchild solution have been smooth by the Born-Infeld parameter [4].

Here, in this paper, we will deal with one of them. The so-called NDL theory [4]. One important assumption in General Relativity is 
that all fields interact in an universal way with gravity. This is the so called Strong Equivalence Principle (SEP). It is well known, with good accuracy, that this is true when it concerns to matter-gravity interaction, i.e., the Weak Equivalence Principle (WEP). But, until now, there is no direct observational confirmation of this assumption to what concerns the gravity-gravity interaction.

In [4], the authors propose an extension of the field-theoretical approach of General Relativity built by $[8,9]$. In their seminal paper [4] the authors reanalyze the Feynman-Deser field-theoretical description and show that its compatibility to General Relativity is not unique. Indeed, as it is shown in [4], one can build a theory in which matter couples universally to gravity in accordance with the Weak Equivalence Principle (WEP), therefore being very similar to General Relativity in which concerns the matter-to-gravity interaction. However, in what concerns the gravity-to-gravity interaction, these theories are in disagreement. Due to the fact that gravity waves are subject to a geometry which is not the same as that of the matter fields, gravitons propagate with different velocity of the photons. Because of the violation of the Strong Equivalence Principle (SEP) and the gravitongraviton self-interaction the NDL theory predicts a massive graviton which propagates with lower velocity than the light.

In a previous work, the authors have shown [10-12] that this theory does not admit a Schwarszchild solution. Later, we also have shown that there is no cosmic string solution in this theory, since the solution is not regular at infinity [13]. In this paper, our main purpose is to proof that the Jebsen-Birkhoff theorem is still valid in the NDL theory. To do that, this manuscript is organized as follows. In the Section 2 we give a brief presentation of the NDL theory based on the original paper [4]. In the Section 3, we write down the detailed calculations to prove the Jebsen-Birkhoff theorem in this framework.

In this paper, our main purpose is to proof that the Jebsen-Birkhoff theorem is still valid in the NDL theory. To do that, this manuscript is organized as follows. In the Section 2 we give a brief presentation of the NDL theory based on the original paper [4]. In the Section 3, we write down the detailed calculations to prove the Jebsen-Birkhoff theorem in this framework and summarizes our main results.

\section{The Born-Infeld Theory: A Special Case}

In this section we summarize the NDL theory following [4,5]. The more detailed presentation is presented in the ref. [4]. To start with, the main lines of the NDL theory are:

- The gravitational interaction is represented by a symmetric tensor $\varphi_{\mu \nu}$ that obeys a nonlinear equation of motion.

- The matter (but not gravity) couples to gravity through the metric $g_{\mu \nu}=\gamma_{\mu \nu}+\varphi_{\mu \nu}$, where $\gamma_{\mu \nu}$ is the flat background metric.

- The self interaction of the gravitational field breaks the universal modification of the space time geometry, i.e., the gravity couples to gravity in a special way distinct from all different forms of energy.

We begin defining the tensor $F_{\alpha \beta \mu}$, which is anti-symmetric in the two first indices, called the gravitational field:

$$
F_{\alpha \beta \mu}:=\frac{1}{2}\left(\varphi_{\mu[\alpha ; \beta]}+F_{[\alpha} \gamma_{\beta] \mu}\right) \text {, }
$$


where $[x, y]=x y-y x$ and the covariant derivative is constructed with the background metric. Indices are raised and lowered with that metric also, and

$$
F_{\alpha}=F_{\alpha \mu \nu} \gamma^{\mu \nu}=\varphi_{, \alpha}-\varphi_{\alpha \mu ; \nu} \gamma^{\mu \nu}
$$

In order to have a nonlinear theory of the gravitational field $F_{\alpha \beta \mu}$ with the correct weak field limit, we assume that the interaction of gravity with itself is described by a functional of $A$ and $B$ which are invariants built from the gravitational field $F_{\alpha \beta \mu}$ :

$$
A=F_{\alpha \beta \mu} F^{\alpha \beta \mu} \text { and } B=F_{\alpha} F^{\alpha} .
$$

In our case we will use the Born-Infeld Lagrangian:

$$
\mathcal{L}=\frac{b^{2}}{k} \sqrt{1-\frac{A-B}{b^{2}}}-1,
$$

where $k$ is the Einstein's constant. Thus, the gravitational action will be:

$$
\mathcal{S}=\int d^{4} x \sqrt{\gamma} \mathcal{L}
$$

where $\gamma$ is the determinant of the Minkowski metric in an arbitrary coordinate system. Taking the variation of the action (3) with respect to the potential $\varphi_{\mu \nu}$, we obtain the following equations of motion:

$$
\left(\mathcal{L}_{U} F^{\lambda(\mu \nu)}\right)_{; \lambda}=-\frac{1}{2} T^{\mu \nu}
$$

where $(x, y)=x y+y x, U=A-B$ and $\mathcal{L}_{U}=\frac{\delta \mathcal{L}}{\delta U}$.

\section{The Non-Static Spherically Symmetric Metric in the NDL Theory: The Vacuum Solution}

We consider here the vacuum exterior region of some spherically symmetric configuration in the NDL theory. In this case our energy momentum tensor will be identically zero in the region outside the matter (a star, for instance). Thus, we begin with the following ansätze:

- The background metric, i.e. $\gamma_{\mu \nu}$, will be the Minkowski metric in spherical coordinates:

$$
d s^{2}=d t^{2}-d r^{2}-r^{2}\left(d \theta^{2}+\sin ^{2} d \phi^{2}\right) .
$$

- The potential $\varphi_{\mu \nu}$ will be :

$$
\varphi_{00}=\mu(r, t) \text { and } \varphi_{11}=-\nu(r, t) .
$$

In a way that our spherically symmetric metric in the NDL theory is:

$$
d s^{2}=(1+\mu(r, t)) d t^{2}-(1+\nu(r, t)) d r^{2}-r^{2}\left(d \theta^{2}+\sin ^{2} d \phi^{2}\right) .
$$

In order to obtain the equations of motion, first we need to compute some elements. The trace and the covariant derivatives of the potential 
are, respectively:

$$
\begin{aligned}
\varphi & =\varphi_{00} \gamma^{00}+\varphi_{11} \gamma^{11}=\mu(r, t)+\nu(r, t), \\
\varphi_{00 ; 0} & =\dot{\mu}, \\
\varphi_{11 ; 1} & =-\nu^{\prime} \\
\varphi_{11 ; 2} & =-\frac{\nu}{r}, \\
\varphi_{11 ; 3} & =-\frac{\nu}{r},
\end{aligned}
$$

where (.) means derivative with respect to $t$ and $\left({ }^{\prime}\right)$ means derivative with respect to $r$. The other components are identically zero. Now, let us compute the non-vanishing components of $F_{\alpha}$. Remind that:

$$
F_{\alpha}=\varphi_{, \alpha}-\varphi_{\alpha \beta ; \sigma} \gamma^{\beta \sigma}
$$

we have:

$$
\begin{aligned}
& F_{0}=\dot{\nu} \\
& F_{1}=\mu^{\prime}-2 \frac{\nu}{r}
\end{aligned}
$$

With these, we can start calculating the field $F_{\alpha \beta \lambda}$. The nonvanishing components are:

$$
\begin{aligned}
F_{022} & =-\frac{1}{2} \dot{\nu} r^{2} \\
F_{033} & =F_{022} \sin ^{2} \theta \\
F_{100} & =-\frac{\nu}{r} \\
F_{122} & =\frac{1}{2}\left(\nu r-\mu^{\prime} r^{2}\right) \\
F_{133} & =F_{122} \sin ^{2} \theta .
\end{aligned}
$$

To write explicitly the Lagrangian we must find the invariants $A$ and $B$ for our particular metric:

$$
\begin{aligned}
A & =-3 \frac{\nu^{2}}{r^{2}}-\left(\mu^{\prime}\right)^{2}+2 \frac{\nu \mu^{\prime}}{r}+(\dot{\nu})^{2} \\
B & =(\dot{\nu})^{2}-\left(\mu^{\prime}-2 \frac{\nu}{r}\right)^{2} \\
U & =A-B=\frac{\nu^{2}}{r^{2}}-2 \frac{\nu \mu^{\prime}}{r}
\end{aligned}
$$

The Lagrangian is given by the expression:

$$
\mathcal{L}=\frac{b^{2}}{k} \sqrt{1-\frac{A-B}{b^{2}}}-1,
$$

and deriving it with respect to $A$, we have:

$$
\mathcal{L}_{A}=-\frac{1}{2 k}\left(1-\frac{A-B}{b^{2}}\right)^{-1 / 2} .
$$

Therefore, the equations of motion are:

$$
\left\{\mathcal{L}_{A} F_{(\mu \nu)}^{\lambda}\right\}_{; \lambda}=0
$$


Computing the non-vanishing components of the covariant derivatives of the gravitational tensor $F^{\lambda \mu \nu}$, we get the following equations:

$$
\begin{aligned}
\mu^{\prime} r-\nu & =0 \\
2 \nu^{3}+b^{2} r^{2} \nu+b^{2} \nu^{\prime} r^{3} & =0 \\
\nu^{2}-b^{2} r^{2} & =0 \\
\ddot{\nu} b^{2} r^{2}+\ddot{\nu} \nu^{2}-\nu \dot{\nu} & =0
\end{aligned}
$$

Replacing (13) into (14), we get:

$$
\nu \dot{\nu}=0,
$$

which means that $\nu$ is a function of $r$ only. Let us now derive (11) with respect to $t$. We have,

$$
\frac{\partial^{2} \mu}{\partial r \partial t}=0
$$

whose integral is $\mu(r, t)=h(r)+f(t)$. Now, let us integrate Equation (12). Equation (12) is a Bernoulli equation. It solution is well-known and can be written as:

$$
\nu(r)=\frac{|C|}{r}\left[1-\left(\frac{r_{0}}{r}\right)^{4}\right]^{-1 / 2},
$$

where $r_{0}^{2}=C / b^{2}, C$ as a constant of integration. This solution is defined only if $r>r_{0}$. Now, integrating (11), we have:

$$
\mu(r, t)=\int \frac{\nu}{r} d r+f(t)
$$

which implies in

$$
\mu(r, t)=|C| \int_{r_{0}}^{r} \frac{d r}{\sqrt{r^{4}-r_{0}^{4}}}+f(t)
$$

whose solution is given in terms of elliptic functions of first kind:

$$
\mu(r, t)=\frac{|C|}{\sqrt{2} r_{0}} F\left[\arccos \left(r_{0} / r\right), 1 / \sqrt{2}\right]+f(t) .
$$

The question now is to determine the function $f(t)$. We remind, however, that for large $r$, this geometry must tend to Minkowski space. Therefore, $f(t)$ must be set as zero in order to satisfy this condition. In this case, we conclude that $\mu$ is also a function of $r$ only:

$$
\mu(r)=\frac{|C|}{\sqrt{2} r_{0}} F\left[\arccos \left(r_{0} / r\right), 1 / \sqrt{2}\right]-F[\pi / 2,1 / \sqrt{2}] .
$$

Our main conclusion is that the Jebsen-Birkhoff theorem in his version $2[14]$ is still valid in the NDL theory of gravity, although this theory does not admit a Schwarszchild solution as showed in refs. [10-12].

\section{Conflicts of Interest}

The authors declare no conflicts of interest regarding the publication of this paper. 


\section{References}

[1] Scherk, J. and Schwarz, J. (1974) Dual Models for Non-Hadrons. Nuclear Physics, Section B, 81, 118-144.

[2] Callan, C.G., Friedan, D., Martinec, E.J. and Perry, M.J. (1985) Strings in Background Fields. Nuclear Physics B, 262, 593-609. https://doi.org/10.1016/0550-3213(85)90506-1

[3] Corda, C. (2009) Interferometric Detection of Gravitational Waves: The Definitive Test for General Relativity. International Journal of Modern Physics D, 18, 2275-2282. arXiv:0905.2505[gr-qc] https://doi.org/10.1142/S0218271809015904

[4] Novello, M., De Lorenci, V.A., de Freitas, L.R. and Aguiar, O.D. (1999) The Velocity of Gravitational Waves. Physics Letters A, 254, 245-250. https://doi.org/10.1016/S0375-9601(99)00080-8

[5] Novello, M. and De Lorenci, V.A. (1997) Do Gravitational Waves Travel at Light Velocity? Annals of Physics, 254, 83-108. https://doi.org/10.1006/aphy.1996.5637

[6] Corlin, A. (1934) A New Hard Component of the Cosmic UltraRadiation. Nature, 133, 63. https://doi.org/10.1038/133063a0

[7] Comelli, D. (2004) Born-Infeld-Type Gravity. Physical Review D, 72, Article ID: 064018. https://doi.org/10.1103/PhysRevD.72.064018

[8] Feynman, R. (1995) Lectures on Gravitation. Addison-Wesley, Boston.

[9] Deser, S. (1970) Self-Interaction and Gauge Invariance. General Relativity and Gravitation, 1, 9-18. https://doi.org/10.1007/BF00759198

[10] Wohlfarth, M.N.R. (2004) Gravity à la Born-Infeld. Classical and Quantum Gravity, 21, 5297. https://doi.org/10.1088/0264-9381/21/8/001

[11] Feigenbaum, J.A., Freund, P.G.O. and Pigli, M. (2004) Gravitational Analogues of Nonlinear Born Electrodynamics. Physical Review D, 57, 4738. https://doi.org/10.1103/PhysRevD.57.4738

[12] Novello, M., Bergliaffa, S.E.P. and Hibberd, K.E. (2004) Analysis of the Static and Spherically-Symmetric Solution in NDL Theory of Gravitation. International Journal of Modern Physics D, 13, 527-537. https://doi.org/10.1142/S0218271804004608

[13] Rosa, T., Guimarães, M. and Neto, J. (2019) On an Exact Cylindrically Symmetric Solution in a Born-Infeld Type Theory of Gravity. Journal of High Energy Physics, Gravitation and Cosmology, 5, 711-718.

[14] Faraoni, V. (2010) The Jebsen-Birkhoff Theorem in Alternative Gravities. Physical Review D, 81, Article ID: 044002. https://doi.org/10.1103/PhysRevD.81.044002 